\title{
Modeling and finite-time sliding model control of a lower limb exoskeleton for use in rehabilitation
}

\author{
Amrapali Khandare · Atharv Itankar · D. S. Kartheek · \\ Uddesh Tople
}

Received: date / Accepted: date

\begin{abstract}
Exoskeleton systems in recent years has become a prime choice technology due to the various possibilities it can deliver. These possibilities comprise the assisting and rehabilitative techniques designed for disabled and elderly people, so that they can regain control of their limbs and in addition to this also to augment and boost the abilities of able-bodied persons during heavy work-load conditions. Many works are reported on the modeling and control of a exoskeleton robot, but very few paper discuss the complete derivation of the model of the system. Here, first the dynamic model of a physical system used as lower limb exoskeleton robot is obtained. Secondly the analysis of the system done that is derived through dynamic modelling of a 3-link robotic manipulator using Euler-Lagrange approach and validation of the corresponding model in simulation. Further, design of a finite-time SMC for desired trajectory tracking of the system is implemented. The dynamic model of the 3-link system and its control using finite-time sliding mode control are validated in MATLAB simulation environment.
\end{abstract}

Keywords Sliding mode control $(\mathrm{SMC}) \cdot$ Exoskeleton $\cdot$ modelling

\section{Introduction}

A lower extremity exoskeleton is a configurable device to be coupled to a person which comprises two leg supports that can attached or coupled to the person's lower limbs [1]. With multiple sensors built within the system, an exoskeleton can estimate user's movement and assist the person's motion accordingly. It applies assistive force / torque to the user's limbs under control, thus providing the mobility as per user requirement. The exoskeleton augments the strength of the user's joints. For example, an exoskeleton facilitates people having movement disorders to get back their ability to stand and walk around [2]. In comparison to conventional physical therapy, exoskeleton assistive rehabilitation will reduce the work of therapists, thereby facilitating more effective and repetitive training, and it is much easier to use for quantitative assessment of the recovery stage by evaluating force and movement patterns [3]. Additionally, exoskeletons can also help a normal person perform heavy tasks.

Exoskeletons are found in various fields due to their advantages of being lightweight and having high load ratios (the ratio of load-mass to body mass), good movement speeds, and efficient energy consumption. Based on the part of the human body to which the exoskeleton is attached, exoskeletons can be classified as specific joint support exoskeletons, upper extremity exoskeletons, lower extremity exoskeletons and full body exoskeletons

Uddesh Tople

VNIT, Nagpur

E-mail: uddesh@students.vnit.ac.in

Mob:- +91-7083107048 
[4]. This paper focuses on the lower extremity exoskeleton for the use in rehabilitation.

Multi-joint exoskeletons are highly nonlinear systems with multi-degree-of freedom, the dynamic modelling for such systems is complex [5]. Problems arise due to lack of sensing, vibration in the joints due to system flexibility, inaccurate positional accuracy and the difficulty in obtaining an accurate model for the system. These challenging topics have gained the attention of a lot of researchers. Berkeley Lower Extremity Exoskeleton (BLEEX) using hydraulic actuators is considered as the first load-carrying exoskeleton[7]. [8] derived the kinematic and dynamic model of a lower extremity knee-joint orthosis and later developed a hybrid controller to repair movements of the knee joint. [9] adopted the Lagrange equation and the assumed mode method to establish the dynamic model of a manipulator with flexible links and joints. [10] developed a dynamic model of flexible manipulators in closed-form which is based on the Newton-Euler equations. A Master-slave controller for an intention-actuated exoskeleton for movement and lower extremity restoration has been developed and implemented effectively [11].

The dynamic model is the sequential representation of the different aspects of system which undergoes change over a period of time. e.g. Position (It represents the state of the robot over its lifetime with respect to some reference). It is a time dependent aspect of the system [5], [6]. Dynamics of a model is related to the forces acting on it and the accelerations produced by it. The two main problems used while deducing the robot dynamics are:

1. Forward dynamics: working on motion from the known internal forces/torques.

2. Inverse dynamics: working on internal forces/ torque from known motion and external forces.

Forward dynamics is also referred as 'direct dynamics' or 'dynamics' main purpose of dynamics is for simulation. Inverse dynamics is the reverse of dynamics. Various applications include on-line control of robot motions and forces, trajectory design and optimization, design of robot mechanisms [12]. Various controllers are designed for the control of lower limb exoskeleton system like Sliding Mode Controller (SMC) [13], adaptive control [15], Lyapunov based control [14], etc. It is knows that SMC is better in uncertainty rejection, remarkable properties of accuracy, robustness, and easy tuning and implementation. Various attempts have been made for implementing the other variantions of SMC controller on exoskeleton [16]. However, most works considered the available dynamic model and very few works reported the dynamic model of the system.

In this paper, a 3-link manipulator body as lower limb extremity exoskeleton is considered and dynamic modelling of the system is obtained. There are two well-known approaches for obtaining the dynamic model of any mechanism, Newton-Euler method which is based on the equilibrium of forces and torques and the EulerLagrange method which is an energy method. In this paper, the Euler-Lagrange method is used for obtaining the dynamic equations of the exoskeleton.

A dynamic model is based on:

1. Space co-ordinates (theta and its derivatives)

2. The forces and torques applied at joints and

3. The model dimensions

Further, the obtained dynamic model is simulated in MATLAB simulation environment and is validated for multiple torque and initial conditions of the angles. A SMC is then implemented to the dynamic model to track its performance in presence of disturbances and the results were studied.

The remaining paper goes like this. Section 2 presents the dynamic modelling of the considered exoskeleton. Dynamic model validation of the exoskeleton is given in Section 3. Design of SMC for the tracking control of the exoskeleton is given in Section 4. Section 5 discuss the results and discussion for the finite time SMC design presented in Section 4. The paper is summarised in Section 6.

\section{Dynamic modelling of lower limb exoskeleton as a tri-link robotic model}

The kinematic model of the hip-knee-ankle system of a lower extremity exoskeleton is depicted in Figure 1. In the figure, the angles $q_{1}, q_{2}, q_{3}$ are considered to be hip, knee and ankle angles, respectively. The dynamic 


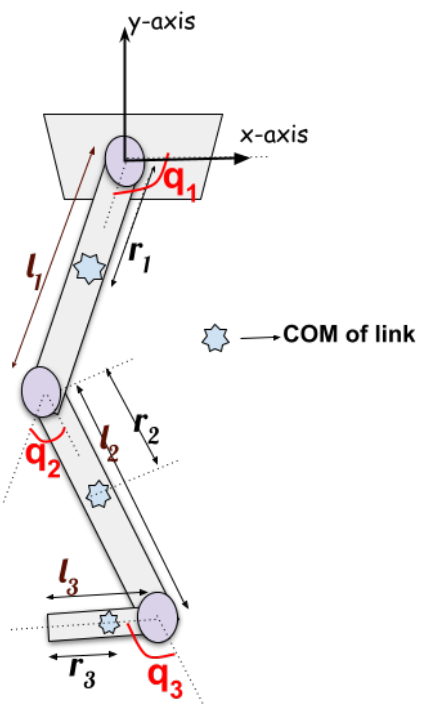

Fig. 1: Generalized pictorial block diagram of an exoskeleton as a tri-link robotic model.

model of the system is derived using Lagrangian formulation[17]. $\mathrm{T}$ is the total kinetic and $\mathrm{P}$ is the potential energy of the lower extremity exoskeleton system. Using T and P the Lagrangian L is defined as

$$
L\left(q_{j}, \dot{q}_{j}\right)=T\left(q_{j}, \dot{q}_{j}\right)-P\left(q_{j}\right)
$$

The Kinetic energy is a function of position and velocity of all links, and Potential energy is dependent on position of the all links i.e angle $q_{j}$. Here, j represents link 1 , link 2 and link 3 of the lower extremity exoskeleton (the upper leg, lower leg and foot) [18]. Using Lagrangian, the dynamic modelling equation of our system is defined as

$$
\begin{gathered}
\frac{d}{d t} \frac{\partial L}{\partial \dot{q_{j}}}-\frac{d L}{\partial q_{j}}+\frac{\partial F}{\partial \dot{q}_{j}}=T_{j} \\
F=\frac{1}{2} c_{1}{\dot{q_{1}}}^{2}+\frac{1}{2} c_{2}\left(\dot{q_{2}}-\dot{q}_{1}\right)^{2}+\frac{1}{2} c_{3}\left(\dot{q_{3}}-\dot{q_{2}}\right)^{2}
\end{gathered}
$$

Where, $F$ is the frictional force term and $c_{1}, c_{2}$ and $c_{3}$ are the coefficients of friction for each joint 1 , joint 2 and joint 3 respectively. $T_{j}$ is the torque. Considering friction into account the dynamic (2) is obtained after the substitution of kinetic energy and potential energy in the Lagrangian equation [19]. The generalized equation for our exoskeleton system is formed as:

$$
D(q) \ddot{q}+V(\dot{q}, q) \dot{q}+G(q)=T
$$

where $\mathrm{q}=\left[q_{1} q_{2} q_{3}\right]^{T}$, is the matrix of the angular motion of hip, knee and ankle of the exoskeleton, respectively, $\mathrm{D} \epsilon \Re^{3 \times 3}$ is the inertial properties matrix, $\mathrm{V} \epsilon \Re^{3 \times 3}$ is the Coriolis and centripetal matrix, $G \epsilon \Re^{3 \times 1}$ is the gravitational vector and $\tau \epsilon \Re^{3 \times 1}$ is the torque value given to the three links, at joint, of the system.

From Eq. (3), $\ddot{q}$ is acquired as,

$$
\ddot{q}=D^{-1}(q)[\tau-V(\dot{q}, q) \dot{q}-G(q)]
$$


Eqn. (5) is expanded as,

$$
\left[\begin{array}{l}
\ddot{q_{1}} \\
\ddot{q_{2}} \\
\ddot{q_{3}}
\end{array}\right]=\left[\begin{array}{lll}
d_{11} & d_{12} & d_{13} \\
d_{21} & d_{22} & d_{23} \\
d_{31} & d_{32} & d_{33}
\end{array}\right]^{-1}\left(-\left[\begin{array}{lll}
v_{11} & v_{12} & v_{13} \\
v_{21} & v_{22} & v_{23} \\
v_{31} & v_{32} & v_{33}
\end{array}\right] \dot{q}-\left[\begin{array}{l}
G_{1} \\
G_{2} \\
G_{3}
\end{array}\right]+\left[\begin{array}{l}
\tau_{1} \\
\tau_{2} \\
\tau_{3}
\end{array}\right]\right)
$$

The parameters of the above equation are;

$$
\begin{aligned}
& d_{11}=m_{1} r_{1}^{2}+m_{2} l_{1}^{2}+m_{2} r_{2}^{2}+m_{3} l_{1}^{2}+m_{3} r_{3}^{2}+m_{3} l_{2}^{2}+2 m_{2} l_{1} r_{2} \cos \left(q_{2}\right) \\
& +2 m_{3} l_{1} l_{2} \cos \left(q_{2}\right)+2 m_{3} l_{2} r_{3} \cos \left(q_{3}\right)+2 m_{3} l_{1} r_{3} \cos \left(q_{2}+q_{3}\right)+I_{z 2}+I_{z 1}+I_{z 3} \\
& d_{12}=m_{2} r_{2}^{2}+m_{3} r_{3}^{2}+m_{3} l_{2}^{2}+m_{3} l_{1} l_{2} \cos \left(q_{2}\right)+m_{3} l_{1} r_{3} \cos \left(q_{2}+q_{3}\right) \\
& +2 m_{3} l_{2} r_{3} \cos \left(q_{3}\right)+m_{2} l_{1} r_{2} \cos \left(q_{2}\right)+I_{z 2}+I_{z 3} \\
& d_{13}=m_{3} r_{3}^{2}+m_{3} l_{2} r_{3} \cos \left(q_{3}\right)+m_{3} l_{1} r_{3} \cos \left(q_{2}+q_{3}\right)+I_{z 3} \\
& d_{21}=m_{2} r_{2}^{2}+m_{3} l_{2}^{2}+m_{3} r_{3}^{2}+m_{2} l_{1} r_{2} \cos \left(q_{2}\right)+m_{3} l_{1} l_{2} \cos \left(q_{2}\right) \\
& +m_{3} l_{1} r_{3} \cos \left(q_{2}+q_{3}\right)+2 m_{3} l_{2} r_{3} \cos \left(q_{3}\right)+I_{z 2}+I_{z 3} \\
& d_{22}=m_{2} r_{2}^{2}+m_{3} l_{2}^{2}+m_{3} r_{3}^{2}+I_{z} 2+2 m_{3} l_{2} r_{3} \cos \left(q_{3}\right)+I_{z 3} \\
& d_{23}=m_{3} r_{3}^{2}+m_{3} l_{2} r_{3} \cos \left(q_{3}\right)+I_{z 3} \\
& d_{31}=m_{3} r_{3}^{2}+m_{3} l_{1} r_{3} \cos \left(q_{3}+q_{2}\right)+m_{3} l_{2} r_{3} \cos \left(q_{3}\right)+I_{z 3} \\
& d_{32}=m_{3} r_{3}^{2}+m_{3} l_{2} r_{3} \cos \left(q_{3}\right)+I_{z 3} \\
& d_{33}=m_{3} r_{3}^{2}+I_{z 3} \\
& v_{11}=-m_{2} l_{1} r_{2} \sin \left(q_{2}\right) \dot{q_{2}}-m_{3} l_{1} l_{2} \sin \left(q_{2}\right) \dot{q_{2}}-m_{3} l_{2} r_{3} \sin \left(q_{3}\right) \dot{q_{3}} \\
& -m_{3} l_{1} r_{3} \sin \left(q_{2}+q_{3}\right) \dot{q}_{2}+b_{1}+b_{2} \\
& v_{12}=-m_{2} l_{2} r_{2} \sin \left(q_{2}\right) \dot{q}_{2}-m_{2} l_{1} r_{2} \sin \left(q_{2}\right) \dot{q}_{1}-m_{3} l_{1} l_{2} \sin \left(q_{2}\right)\left(\dot{q}_{1}+\dot{q}_{2}\right) \\
& -m_{3} l_{2} r_{3} \sin \left(q_{3}\right) \dot{q}_{3}-m_{3} l_{1} r_{3} \sin \left(q_{2}+q_{3}\right)\left(\dot{q}_{1}+\dot{q}_{2}+\dot{q}_{3}\right)-b_{2} \\
& v_{13}=-m_{3} l_{1} r_{3} \sin \left(q_{2}+q_{3}\right) \dot{q}_{2}-m_{3} l_{2} r_{3} \sin \left(q_{3}\right)\left(\dot{q_{1}}+\dot{q}_{2}+\dot{q}_{3}\right) \\
& v_{21}=m_{2} l_{1} r_{2} \sin \left(q_{2}\right) \dot{q}_{1}+m_{3} l_{1} l_{2} \sin \left(q_{2}\right) \dot{q}_{1}+m_{3} l_{1} r_{3} \sin \left(q_{2}+q_{3}\right) \dot{q}_{1}-2 m_{3} l_{2} r_{3} \sin \left(q_{3}\right) \dot{q}_{3}-b_{2} \\
& v_{22}=-2 m_{3} l_{2} r_{3} \sin \left(q_{3}\right) \dot{q}_{3}+b_{2}+b_{3} \\
& v_{23}=-m_{3} l_{2} r_{3} \sin \left(q_{3}\right) \dot{q}_{3}-b_{3} \\
& v_{31}=m_{3} l_{1} r_{3} \sin \left(q_{2}+q_{3}\right) \dot{q}_{1}+m_{3} l_{2} r_{3} \sin \left(q_{3}\right)\left(\dot{q}_{1}+\dot{q}_{2}\right) \\
& v_{32}=m_{3} l_{2} r_{3} \sin \left(q_{3}\right)\left(\dot{q}_{1}+\dot{q}_{2}\right)-b_{3} \\
& v_{33}=-b_{3} \\
& G_{1}=m_{1} g r_{1} \cos \left(q_{1}\right)+m_{2} g l_{1} \cos \left(q_{1}\right)+m_{2} g r_{2} \cos \left(q_{1}+q_{2}\right)+m_{3} g l_{1} \cos \left(q_{1}\right) \\
& +m_{3} g l_{2} \cos \left(q_{1}+q_{2}\right)+m_{3} g r_{3} \cos \left(q_{1}+q_{2}+q_{3}\right) \\
& G_{2}=m_{2} r_{2} g \cos \left(q_{1}+q_{2}\right)+m_{3} g l_{2} \cos \left(q_{1}+q_{2}\right)+m_{3} g r_{3} \cos \left(q_{1}+q_{2}+q_{3}\right) \\
& G_{3}=m_{3} g r_{3} \cos \left(q_{1}+q_{2}+q_{3}\right)
\end{aligned}
$$

\section{Dynamic Modelling Validation}

The proposed dynamic model of lower extremity exoskeleton was simulated on MATLAB software. The following parameters, taking into account practical aspects, were considered. The exoskeleton links are taken as uniform rods. Moment of inertia is taken about centre of each rod. Here three cases are considered for the model validation. 


\begin{tabular}{|c|c|c|c|c|c|c|c|}
\hline Parameter & Variable & Value & Unit & Parameter & Variable & Value & Unit \\
\hline Mass & $m_{1}$ & 2.75 & $\mathrm{~kg}$ & Moment of Inertia & $I_{z 1}$ & 0.012 & $\mathrm{kgm}^{2}$ \\
& $m_{2}$ & 2.75 & $\mathrm{~kg}$ & & $I_{z 2}$ & 0.0038 & $\mathrm{kgm}^{2}$ \\
& $m_{3}$ & 0.25 & $\mathrm{~kg}$ & & $I_{z 3}$ & 0.000208 & $\mathrm{kgm}^{2}$ \\
\hline Length & $l_{1}$ & 0.23 & $\mathrm{~m}$ & Frictional torque coefficient & $b_{1}$ & 0.7056 & $\mathrm{Nms}$ \\
& $l_{2}$ & 0.13 & $\mathrm{~m}$ & & $b_{2}$ & 0.7056 & $\mathrm{Nms}$ \\
& $l_{3}$ & 0.1 & $\mathrm{~m}$ & & $b_{3}$ & 0.7056 & $\mathrm{Nms}$ \\
\hline Distance & $r_{1}$ & 0.115 & $\mathrm{~m}$ & & & & \\
to centre & $r_{2}$ & 0.065 & $\mathrm{~m}$ & & & & \\
of mass & $r_{3}$ & 0.05 & $\mathrm{~m}$ & & & & \\
\hline
\end{tabular}

Table 1: Parameters considered during simulation

Case 1 Initial condition (ICs) with respect to positive $\mathrm{X}$-axis for each link is taken as $0 \mathrm{rad}$, and torque $\tau=0$ is applied to the system.

For observing the free motion behavior of our lower extremity exoskeleton which is a nonlinear system, the three angles are set to zero and torque is also set to zero. As shown in the Fig 2, due to the effects of gravitational force and frictional force, all the three links are stabilized slowly at the position pointing to the ground after damping oscillations.

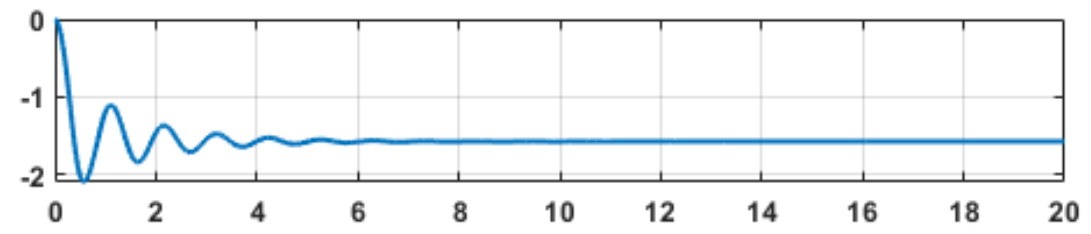

(a) $q_{1}$ (rad) vs time(sec)

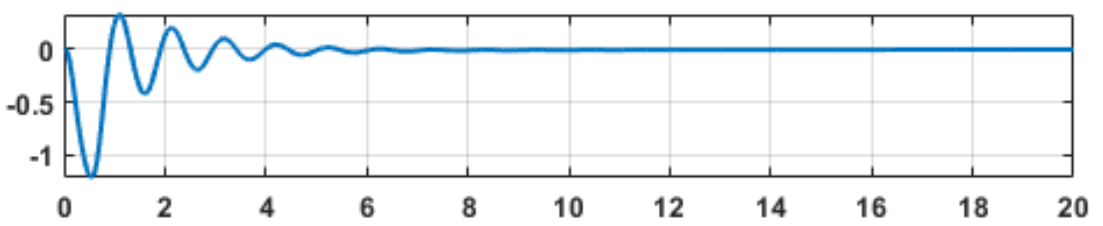

(b) $q_{2}$ (rad) vs time(sec)

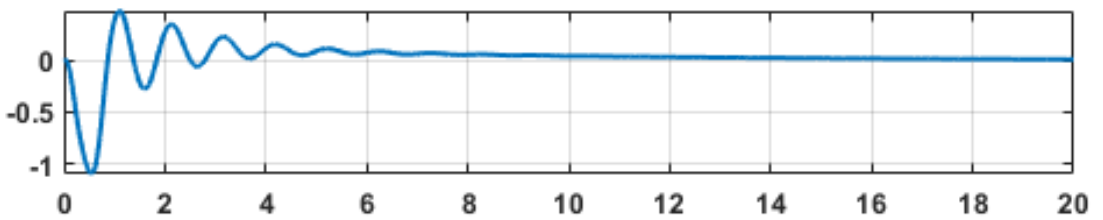

(c) $q_{3}$ (rad) vs time(sec)

Fig. 2: Angular position $\mathrm{q}(\mathrm{rad})$ response with respect to time $\mathrm{t}(\mathrm{secs})$

Case 2 Initial condition with respect to positive $x$-axis for $q_{1}=0.5 \mathrm{rad}, q_{2}=1 \mathrm{rad}, q_{3}=-0.5 \mathrm{rad}$ and $\tau=0$ 
ie.e zero torque is applied. The angular positions in this case is presented in Fig. 3. It is very apparent from Fig. 3 that the angular positions settled to the value to its initial condition value. There is influence of torque, only initial conditions value is there.

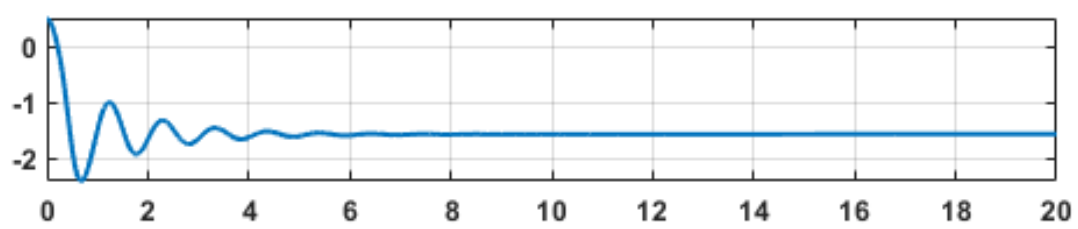

(a) $q_{1}$ (rad) vs time(sec)

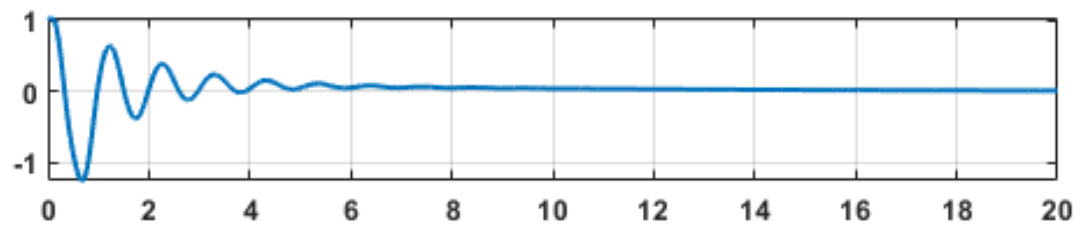

(b) $\mathrm{q}_{2}$ (rad) vs time(sec)

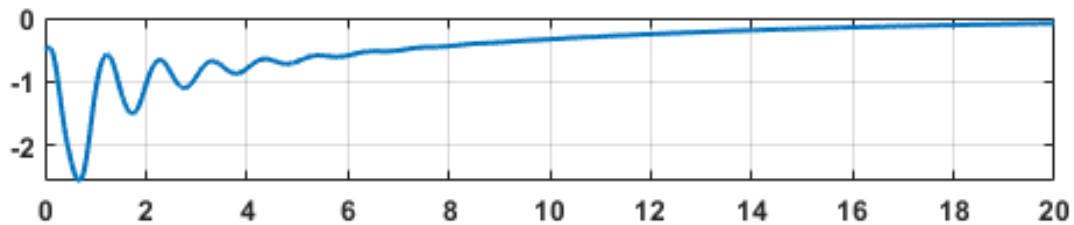

(c) $q_{3}$ (rad) vs time(sec)

Fig. 3: Angular position $\mathrm{q}(\mathrm{rad})$ response with respect to time $\mathrm{t}(\mathrm{secs})$

Case 3 Initial condition with respect to positive $\mathrm{X}$-axis is taken as zero for each link.

Constant torque is applied at respective joint $T_{1}=7 \mathrm{Nm}, T_{2}=0.8 \mathrm{Nm}, T_{3}=0.02 \mathrm{Nm}$ With application of constant torque on active joints. The results in this case is presented in Fig. 4. It is apparent from Fig. 4 that each of the three links is stabilized slowly at a position where the applied torque balances the torque due to gravity.

Case 4 The initial condition with respect to positive $\mathrm{X}$-axis is taken as $0.5 \mathrm{rad}$ for each angle, i.e $q_{i}(o)=$ $(0,0,0)^{T}$ and the torque value is applied as given by $T_{1}=7 \mathrm{Nm}, T_{2}=0.8 \mathrm{NM}, T_{3}=0.02 \mathrm{Nm}$ to respective link. The results in this case is presented in Fig. 5. It is apparent from Fig. 5 that each of the three links is settled slowly at a position where the applied torque balances the torque due to gravity and initial values. It is apparent from Figs. 2 to 5 that the dynamic model proposed in the Section 2 is validated successfully in the simulation environment.

\section{Sliding Mode Controller of the lower limb exoskeleton}

In this section, Sliding Mode Control strategy for exoskeleton model is designed. SMC is fundamentally resistant to changes in the parameters, external disturbances and uncertainty. The aim for controller design is the convergence and tracking of the angular positions defined by joint angle, $q_{1}$ (hip), $q_{2}$ (knee) and $q_{3}$ (ankle) with respect to the desired signal in the presence of bounded but unknown external disturbances [20].

A closed loop control system block diagram for 3 link exoskeleton is shown in fig. 6 . The following equation 


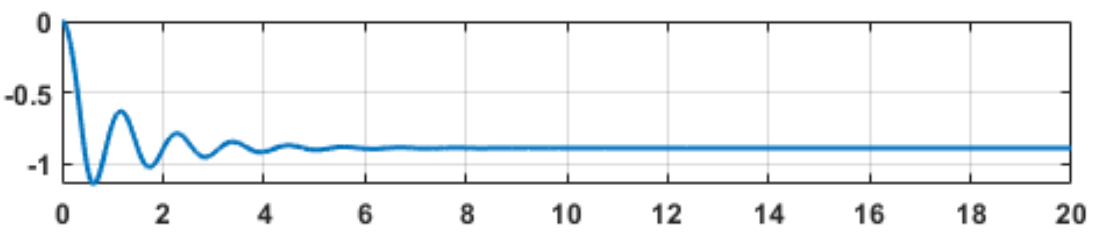

(a) $q_{1}$ (rad) vs time(sec)

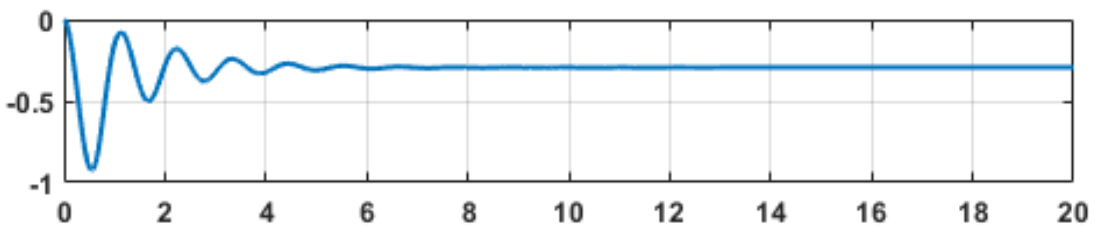

(b) $q_{2}$ (rad) vs time(sec)

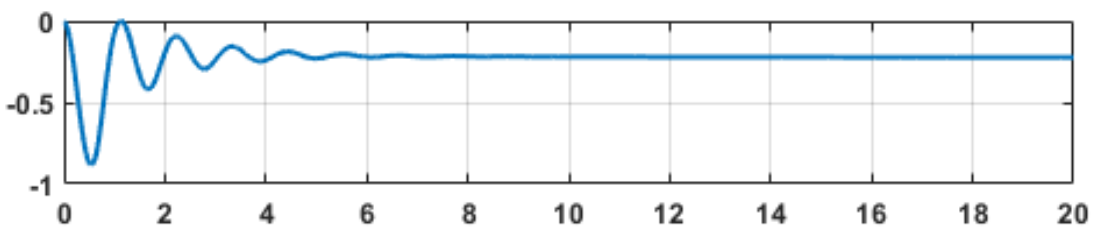

(c) $q_{3}$ (rad) vs time(sec)

Fig. 4: Angular position $\mathrm{q}(\mathrm{rad})$ response with respect to time $\mathrm{t}(\mathrm{secs})$ when $q_{i}(0)=(0,0,0) T_{1}=7 \mathrm{Nm}, T_{2}=0.8$ $\mathrm{Nm}, T_{3}=0.02 \mathrm{Nm}$

represents exoskeleton model (5) in state-space form.

$$
\begin{gathered}
\dot{q}_{1}=q_{2} \\
\ddot{q}_{1}=\dot{q}_{2}=f+A \tau
\end{gathered}
$$

The tracking angular position error and velocity error are defined as follows,

$$
\begin{aligned}
e_{1} & =q_{d}-q_{1} \\
e_{2}=\dot{e}_{1} & =\dot{q}_{d}-q_{2}
\end{aligned}
$$

The sliding surface $s$ which enforces sliding mode is given by

$$
\begin{aligned}
s & =e_{2}+c * e_{1} \\
\dot{s} & =\dot{e_{2}}+c * e_{2} \\
f & =D^{-} 1(-V \dot{q}-G) \\
A & =D^{-1} \\
\ddot{q} & =D^{-} 1(q)[\tau-V(\dot{q}, q) \dot{q}-G(q)] \\
\tau=u_{\text {joint }} & =-D *\left(-c * e_{2}-\dot{q}_{d}+f-k * \operatorname{sgn}(s)\right)
\end{aligned}
$$

Where $u_{\text {joint }}=\tau=\left[\tau_{1}, \tau_{2}, \tau_{3}\right]$, $\mathrm{c}$ is positive integer, $\operatorname{sgn}()$ is the signum function, ' $\mathrm{k}$ ' is the switching gain which is inversely proportional to the reaching time from initial position to the desired position. 


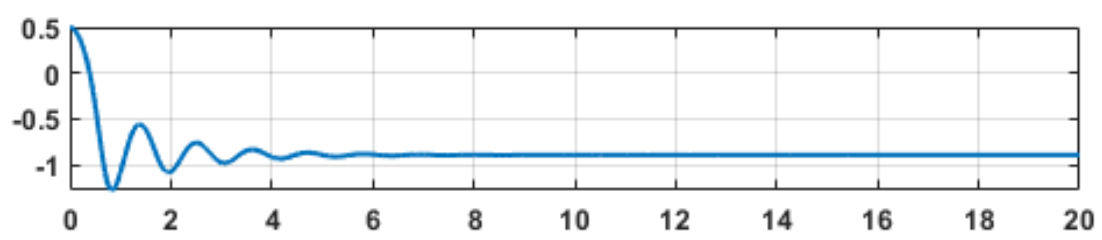

(a) $q_{1}($ rad) vs time(sec)

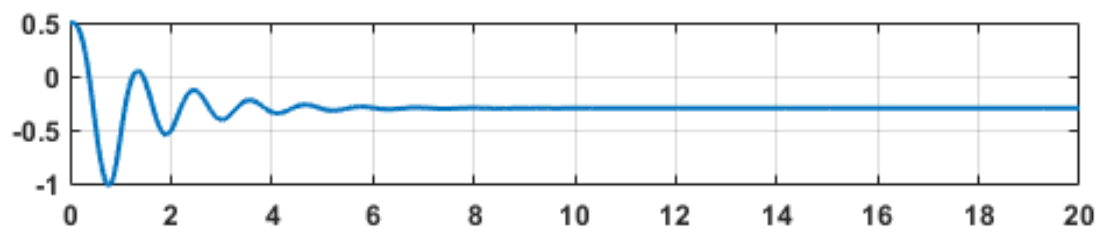

(b) $q_{2}$ (rad) vs time(sec)

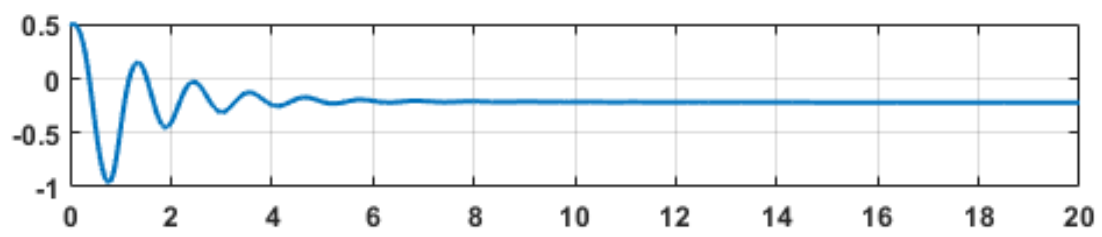

(c) $q_{3}(\mathrm{rad})$ vs time $(\mathrm{sec})$

Fig. 5: Angular position q(rad) response with respect to time t(secs) when $q_{i}(o)=(0,0,0)^{T}$ and $T_{1}=7 \mathrm{Nm}$, $T_{2}=0.8 \mathrm{NM}, T_{3}=0.02 \mathrm{Nm}$

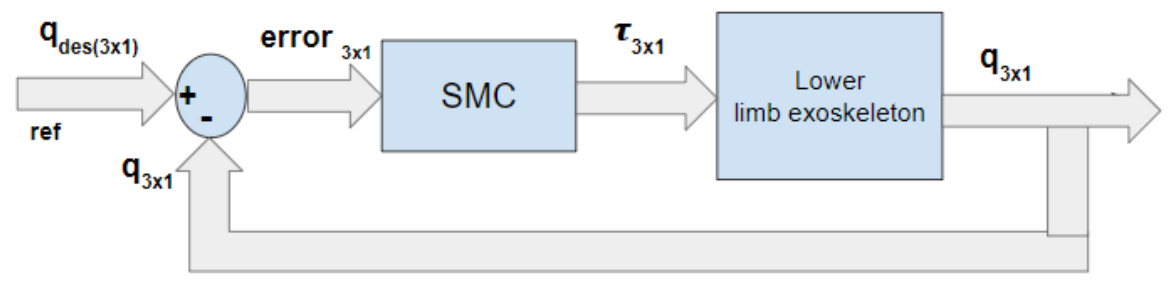

Fig. 6: Block diagram

\subsection{Finite time SMC}

The fault with classic SMC is that it can only achieve asymptotic stability meaning that the tracking error reduces to zero as time reaches to infinity. Finite time control aims to provide excellent properties, such as faster convergence rate, higher accuracy, better robustness against uncertainties and disturbance rejection property [18]. Classic SMC suffers from high frequency chattering, as the infinite switching frequency required by ideal sliding mode is not obtainable practically [22].

Let us introduce a sliding surface in state space of the system as

$$
\sigma=e_{2}+c * e_{1}
$$


In order to achieve asymptotic convergence of $q_{1}$ and $q_{2}$ to zero, in presence of bounded disturbances, we have to drive $\sigma$ to zero in finite time with using control $\tau$. To achieve this, we apply the Lyapunov function techniques. Let us consider our semi-definite Lyapunov function candidate be in the form

$$
V=\frac{1}{2} \sigma^{2}
$$

In order to provide asymptotic stability following conditions must be satisfied.

$$
\begin{aligned}
\dot{V} & <0 \text { for } \sigma \neq 0 \\
\lim _{|\sigma| \rightarrow \infty} V & =\infty
\end{aligned}
$$

To achieve finite time convergence, 1 st condition is modified to,

$$
\dot{V} \leq-\alpha V^{\frac{1}{2}}, \alpha>0
$$

Integrating over time interval $0 \leq \tau \leq t$, we get

$$
V_{1 / 2}(t) \leq-\frac{1}{2} \alpha t+V^{1 / 2}(0)
$$

$V(t)$ will reach finite time $t_{r}$ bounded by

$$
t_{r} \leq \frac{2 V^{1 / 2}(0)}{\alpha}
$$

Therefore, a control $u$ that is to be computed to satisfy equation 13. that will drive the variable $\sigma$ to zero in finite time and will keep it at zero thereafter.

The derivative of $\mathrm{V}$ is calculated as

$$
\dot{V}=\sigma \dot{\sigma}=\sigma\left(c q_{2}+f\left(q_{1}, q_{2}, t\right)+u\right)
$$

Assuming $u=-c q_{2}+v$ and substituting it into Eq.(16) we get

$$
\left.\dot{V}=\sigma\left(f\left(q_{1}, q_{2}, t\right)+v\right)=\sigma f\left(q_{1}, q_{2}, t\right)+\sigma v\right) \leq|\sigma| L+\sigma v
$$

Selecting $v=-k \operatorname{sign}(\sigma)$ where

and

$$
\operatorname{sign}(q)=\left\{\begin{array}{r}
1 \text { if } q>0 \\
-1 \text { if } q<0
\end{array}\right.
$$

$$
\operatorname{sign}(0) \epsilon[-1,1]
$$

with $k>0$ and putting it into eq. (17), we obtain

$$
\dot{V} \leq|\sigma| L-|\sigma| k=-|\sigma|(k-L)
$$

Considering eq. (11), condition (12) can be rewritten as

$$
\dot{V} \leq-\alpha V^{1 / 2}=-\frac{\alpha}{\sqrt{2}}|\sigma|, \alpha>0
$$

Combining Eqs. (20) and (21) we obtain

$$
\dot{V} \leq-|\sigma|(\rho-L)=-\frac{\alpha}{\sqrt{2}}|\sigma|
$$

Finally, the control gain $\rho$ is ontained as

$$
k=L+\frac{\alpha}{\sqrt{2}}
$$

This value of $\mathrm{k}$ is in control equation given in (9). 


\section{Results and discussion for finite time SMC control of the exoskeleton robot}

In this section, the simulations results of the performance of SMC for the exoskeleton robot is presented. Here two cases are considered for the results validation.

Case 1 When the $q_{d}$ (desired position) $=1(\mathrm{rad})$ for each link without addition of any disturbances.

The simulation results for angular positions and torque requirements are shown in Fig. 7 and 8, respectively. The SMC controller is responsible for minimizing the error in position and subsequently brings it to zero with application of variable torque. It is observed from Fig. 8 that the torque remains constant to maintain the system at the desired position.
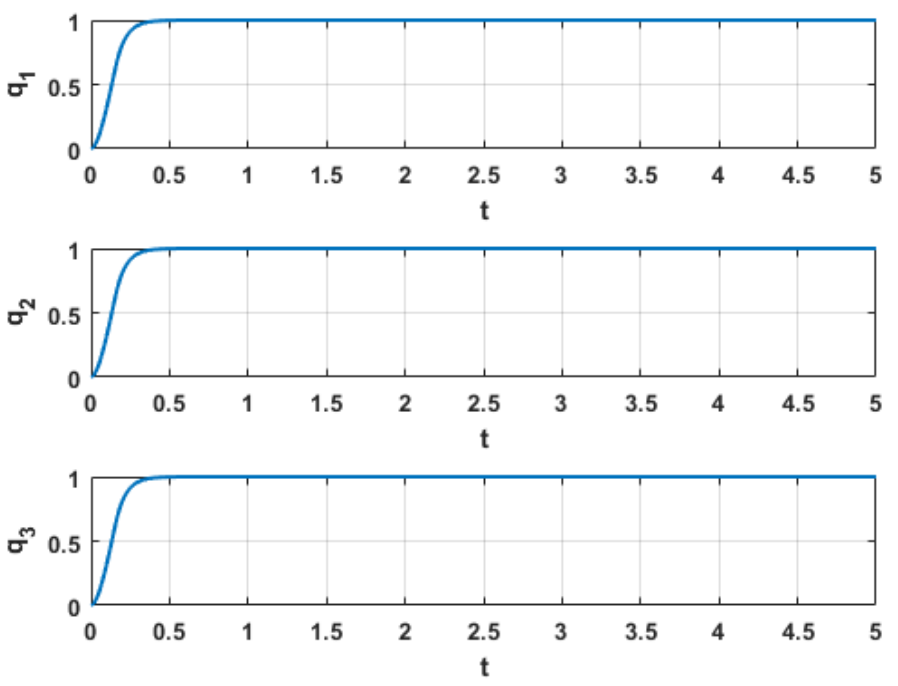

Fig. 7: Angular position $\mathrm{q}(\mathrm{rad})$ response with respect to time $\mathrm{t}(\mathrm{secs})$, when the $q_{d}$ (desired position) $=1(\mathrm{rad})$ for each link without addition of any disturbances

Case 2 When $q_{d}=1(\mathrm{rad})$ for each link and disturbance $=20 \sin (\mathrm{t})$ applied from $\mathrm{t}=2.5 \mathrm{sec}$ to $\mathrm{t}=3 \mathrm{sec}$ to each joints positions. The simulation results for angular positions and torque requirements are shown in Fig. 9 and 10 , respectively.

When an external disturbance is applied to the joint positions, there is a change in angle position so the controller changes the torque accordingly to force the system to regain its desired value. This shows the robust quality of SMC controller.

\section{Conclusions}

In this paper, finite time sliding mode controller is proposed. A pivotal feature of the proposed method is that the tracking errors is forced to settle at zero after a desired finite time in the presence of external disturbance and uncertainties. We performed a elaborate literature survey on exoskeleton; types of exoskeletons; history of exoskeletons and aspects of mechanical design in the current existing models of exoskeletons. We designed the dynamic modelling of the system using Euler-Lagrange method. Dynamic model equations are derived in presence of external disturbances. Validation of the proposed modeling is carried out in MATLAB software and is experimented for multiple values of torque and initial conditions of the limb-angles. The Finite Time 

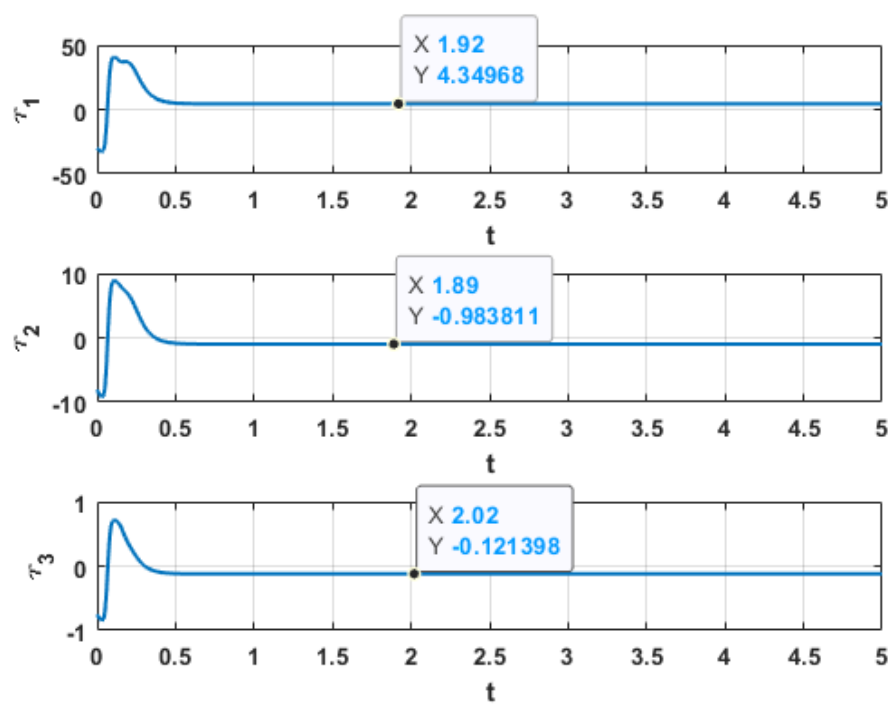

Fig. 8: Response of Torque $(\mathrm{Nm})$ vs time $(\mathrm{sec})$ when the $q_{d}$ (desired position) $=1(\mathrm{rad})$ for each link without addition of any disturbances
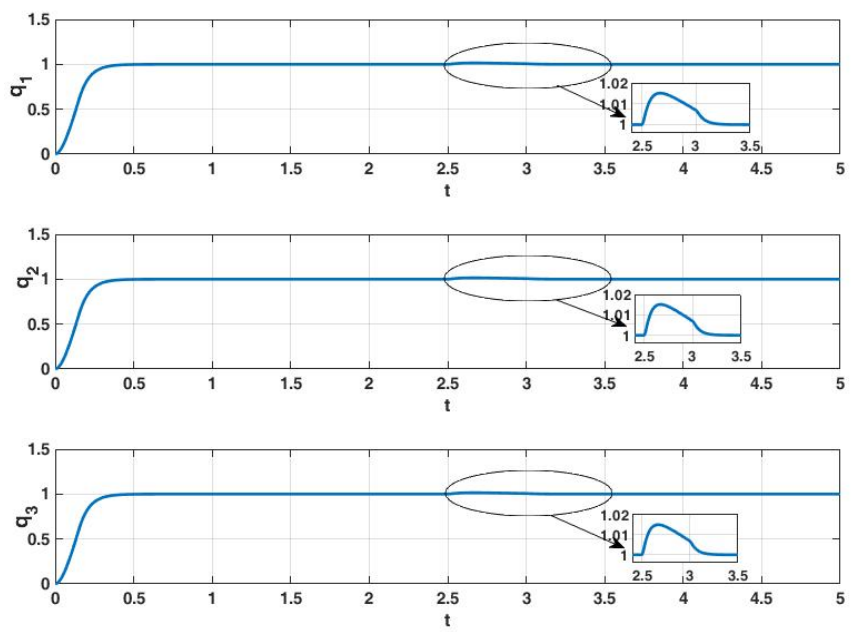

Fig. 9: Angular position $\mathrm{q}(\mathrm{rad})$ response with respect to time $\mathrm{t}(\mathrm{secs})$ with disturbance

control strategy is used for the controller design and it's effectiveness is tested through various simulations. The proposed controller is found to be highly robust against system parameters changes. The future work includes implementation of higher order sliding mode controller. 

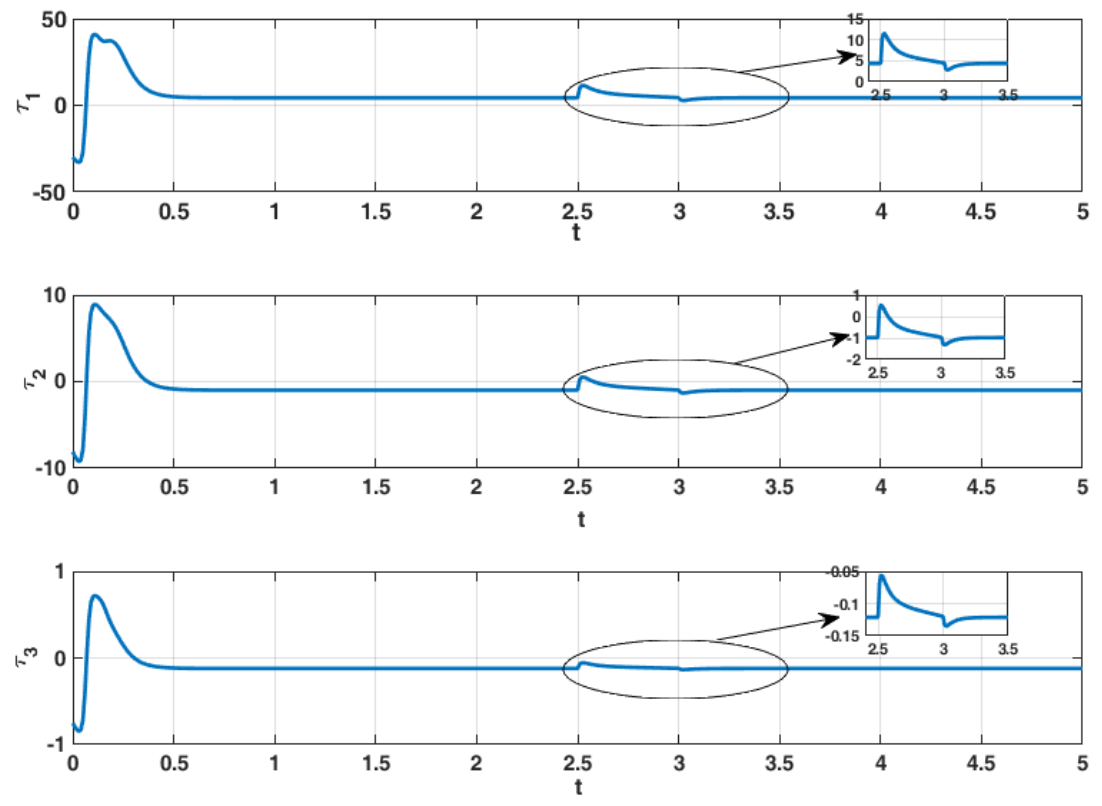

Fig. 10: Response of Torque(Nm) vs time(sec) with disturbance

\section{References}

1. M. F. Hamza, R. A. R. Ghazilla, B. B. Muhammad, and H. J. Yap, "Balance and stability issues in lower extremity exoskeletons: A systematic review," Biocybern. Biomed. Eng., vol. 40, no. 4, pp. 1666-1679, 2020, doi: 10.1016/j.bbe.2020.09.004.

2. B. Wang, Y. Liang, D. Xu, Z. Wang, and J. Ji, "Design on electrohydraulic servo driving system with walking assisting control for lower limb exoskeleton robot,” Int. J. Adv. Robot. Syst., vol. 18, no. 1, pp. 1-17, 2021, doi: 10.1177/1729881421992286.

3. A. M. Dollar and H. Herr, "Lower extremity exoskeletons and active orthoses: Challenges and state-of-the-art," IEEE Trans. Robot., vol. 24, no. 1, pp. 144-158, 2008, doi: 10.1109/TRO.2008.915453.

4. T. Eiammanussakul and V. Sangveraphunsiri, "A lower limb rehabilitation robot in sitting position with a review of training activities," J. Healthc. Eng., vol. 2018, 2018, doi: 10.1155/2018/1927807.

5. R. M. Andrade and P. Bonato, "The Role Played by Mass, Friction, and Inertia on the Driving Torques of Lower-Limb Gait Training Exoskeletons," IEEE Trans. Med. Robot. Bionics, vol. 3, no. 1, pp. 125-136, 2021, doi: 10.1109/tmrb.2021.3052014.

6. S. Glowinski, M. Obst, S. Majdanik, and B. Potocka-Banaś, "Dynamic model of a humanoid exoskeleton of a lower limb with hydraulic actuators," Sensors, vol. 21, no. 10, pp. 1-20, 2021, doi: 10.3390/s21103432.

7. A. Zoss, H. Kazerooni, and A. Chu, "On the mechanical design of the Berkeley Lower Extremity Exoskeleton (BLEEX)," 2005 IEEE/RSJ Int. Conf. Intell. Robot. Syst. IROS, pp. 3465-3472, 2005, doi: 10.1109/IROS.2005.1545453.

8. S. Mefoued, S. Mohammed, and Y. Amirat, "Knee joint movement assistance through robust control of an actuated orthosis," IEEE Int. Conf. Intell. Robot. Syst., pp. 1749-1754, 2011, doi: 10.1109/IROS.2011.6048534.

9. B. Subudhi and A. S. Morris, "Dynamic modelling, simulation and control of a manipulator with flexible links and joints," Robotics and Autonomous Systems, vol. 41, no. 4, pp. 257-270, (2002).

10. L. Bascetta, G. Ferretti, and B. Scaglioni, "Closed form Newton-Euler dynamic model of flexible manipulators," Robotics, vol. 35, no. 5, pp. 1006-1030, (2017).

11. Huang, G., Zhang, W., Meng, F., Zhangguo, Y., Chen, X., Ceccarelli, M., et al. Master-slave control of an intention-actuated exoskeleton robot for locomotion and lower extremity rehabilitation. International Journal of Precision Engineering and Manufacturing, 19(7), 983-991. (2018)

12. J. Wei, D. Cao, L. Liu, and W. Huang, "Global mode method for dynamic modeling of a flexible-link flexible-joint manipulator with tip mass," Applied Mathematical Modelling, vol. 48, pp. 787-805, (2017).

13. Y. Tu et al., "An adaptive sliding mode variable admittance control method for lower limb rehabilitation exoskeleton robot," Appl. Sci., vol. 10, no. 7, 2020, doi: 10.3390/app10072536.

14. F. Qin, H. Zhao, S. Zhen, and Y. Zhang, "Adaptive robust control for lower limb rehabilitation robot with uncertainty based on Udwadia-Kalaba approach,” Adv. Robot., vol. 34, no. 15, pp. 1012-1022, 2020, doi: 10.1080/01691864.2020.1774414. 
15. D. Shi, W. Zhang, W. Zhang, L. Ju, and X. Ding, "Human-centred adaptive control of lower limb rehabilitation robot based on human-robot interaction dynamic model," Mech. Mach. Theory, vol. 162, p. 104340, 2021, doi: 10.1016/j.mechmachtheory.2021.104340.

16. J. Zhou, S. Yang, and Q. Xue, "Lower limb rehabilitation exoskeleton robot: A review," Adv. Mech. Eng., vol. 13, no. 4, pp. 1-17, 2021, doi: 10.1177/16878140211011862.

17. A. S. Nair and D. Ezhilarasi, "Performance Analysis of Super Twisting Sliding Mode Controller by ADAMS-MATLAB Cosimulation in Lower Extremity Exoskeleton,” Int. J. Precis. Eng. Manuf. - Green Technol., vol. 7, no. 3, pp. 743-754, 2020, doi: 10.1007/s40684-020-00202-w.

18. M. W. Spong, S. Hutchinson, M. Vidyasagar. . Robot dynamics and control, 2nd edn. Springer.(2004)

19. Y. Li, L. Yan, H. Qian, J. Wu, S. Men, and N. Li, "Dynamics and kinematics analysis and simulation of lower extremity power-assisted exoskeleton," J. Vibroengineering, vol. 16, no. 2, pp. 781-791, 2014.

20. Saad M., Tople U., Khandare A., Ul Islam Z. Robust Sliding Mode Controller (RSMC) for an Omniwheeled Mobile Robot with Uncertainties and External Perturbations. In: Kalamkar V., Monkova K. (eds) Advances in Mechanical Engineering. Lecture Notes in Mechanical Engineering. Springer, Singapore.(2021)

21. W. Van Dijk and H. Van Der Kooij, “XPED2: A passive exoskeleton with artificial tendons," IEEE Robot. Autom. Mag., vol. 21, no. 4, pp. 56-61, 2014, doi: 10.1109/MRA.2014.2360309.

22. R. Bkekri, A. Benamor, M. A. Alouane, G. Fried, and H. Messaoud, "Robust adaptive sliding mode control for a human-driven knee joint orthosis," Ind. Rob., vol. 45, no. 3, pp. 379-389, 2018, doi: 10.1108/IR-11-2017-0205.

Data Availability : Data sharing not applicable to this article as no datasets were generated or analysed during the current study.

\section{Compliance with Ethical Standards}

Conflict of interest : The authors declare that they have no conflict of interest. 